\title{
GAMMA PROCESSES AND FINITE TIME SURVIVAL PROBABILITIES
}

\author{
By David C. M. Dickson and Howard R. Waters \\ Heriot-Watt University, Edinburgh
}

\begin{abstract}
In this paper we derive formulae for finite time survival probabilities when the aggregate claims process is a Gamma process. We illustrate how a compound Poisson process can be approximated by a Gamma process and by a process defined as a translated Gamma process. We also show how survival probabilities for a compound Poisson process can be approximated by those for a Gamma process or a translated Gamma process.
\end{abstract}

\section{KEYWORDS}

Gamma process; finite time; survival probability.

\section{INTRODUCTION}

The Gamma process was introduced into the actuarial literature by DUFRESNE, Gerber and SHIU [1991]. They defined a Gamma process as a limit of compound Poisson processes and proposed it as a model for the aggregate claims process. They discussed many of the properties of this process and, in particular, showed how to calculate the probability of ultimate ruin for such a process. This paper takes this study a stage further and is concerned mainly with the probability of ruin/survival in finite time.

In the next section we derive (very simple) formulae for the probability of survival in finite time for a Gamma process. In $\S 3$ we show how to approximate a compound Poisson process by a Gamma process and investigate numerically how well the probability of survival in finite time for the former process is approximated by that for the latter process. The advantage in approximating a compound Poisson process in this way is that, as we show in $\S 2$, the probability of survival in finite time for a Gamma process is particularly easy to calculate. In $\S 4$ we introduce what we term a translated Gamma process and we carry out an investigation similar to that in $\S 3$.

\section{FINITE TIME SURVIVAL FOR THE GAMMA PROCESS}

The main result in this section gives formulae for the probability of survival in finite time for a Gamma process. These formulae are derived from standard formulae for a compound Poisson process. Before deriving these results we show how to define/construct a (standardised) Gamma process as a limit of 
compound Poisson processes. This construction follows precisely the method outlined by DuFresne et al. [1991, $\$ \S 2$ and 3]. However, we provide more details of the construction since some of these details are important for the proof of the main result.

For $x>0$ define the function $Q(x)$ as follows:

$$
Q(x)=\int_{x}^{\infty} \frac{\exp \{-y\}}{y} d y
$$

(This is precisely the same as the function $Q(x)$ in DUFRESNE et al. [1991, $\S 3]$ with their parameters $a$ and $b$ both taken to be 1.)

Now let $\{S(t ; 1)\}_{t>0}$ be a compound Poisson process such that:

(a) the number of claims occurring in $(0, t]$ has a Poisson distribution with mean $t Q(1)$, and,

(b) individual claims amounts have a distribution function $P(y ; 1)$, where:

$$
\begin{aligned}
P(y ; 1) & =0 & & \text { for } \quad y<1 \\
& =[Q(1)-Q(y)] / Q(1) & & \text { for } \quad y \geq 1 .
\end{aligned}
$$

For $n=2,3, \ldots$, define the process $\{S(t ; n)\}_{t}>0$ as follows:

$$
S(t ; n)=S(t ; n-1)+X_{n}(t)
$$

where $\left\{X_{n}(t)\right\}_{t>0}$ is a compound Poisson process, independent of $\{S(t ; n-1)\}_{t>0}$, such that:

(c) the number of claims occurring in $(0, t]$ has a Poisson distribution with mean $t[Q(1 / n)-Q(1 /(n-1))]$, and,

(d) individual claim amounts have a distribution function $I(y ; n)$, where:

$$
\begin{aligned}
\Pi(y ; n) & =0 & & \text { for } \quad y<1 / n \\
& =\frac{Q(1 / n)-Q(y)}{Q(1 / n)-Q(1 /(n-1))} & & \text { for } \quad 1 / n \leq y<1 /(n-1) \\
& =1 & & \text { for } \quad y \geq 1 /(n-1)
\end{aligned}
$$

Then it is easy to show that for $n=1,2,3, \ldots\{S(t ; n)\}_{t>0}$ is a compound Poisson process such that:

(e) the number of claims occurring in $(0, t]$ has a Poisson distribution with mean $t Q(1 / n)$, and,

(f) individual claims amounts have a distribution function $P(y ; n)$, where:

$$
\begin{aligned}
P(y ; n) & =0 & & \text { for } \quad y<1 / n \\
& =[Q(1 / n)-Q(y)] / Q(1 / n) & & \text { for } \quad y \geq 1 / n
\end{aligned}
$$

(Note that $S(t ; n)$ and $P(y ; n)$ in this paper were denoted $S(t ; x)$ and $P(y ; x)$, respectively, with $x=1 / n$ by DUFRESNE et al. [1991].) 
The standardised Gamma process is the process $\left\{S_{S G}(t)\right\}_{t>0}$ defined by:

$$
S_{S G}(t)=\operatorname{Lim}_{n \rightarrow \infty} S(t ; n) \text { for } t>0
$$

It is important to note that this limit exists surely, rather than just almost surely, for each $t>0$, since, from $(2.1), S(t ; n)$ is monotonic non-decreasing as $n \rightarrow \infty$.

Since $S(t ; n)$ converges surely to $S_{S G}(t)$, it also converges in distribution. However, DUfRESNE et al. [1991, §3] show that $S(t ; n)$ converges in distribution to a random variable with a Gamma $(t, 1)$ distribution. Hence, $S_{S G}(t)$ has a $\operatorname{Gamma}(t, 1)$ distribution. Finally in this construction/definition, for any $\alpha>0$ and $\beta>0$, define a new stochastic process $\left\{S_{G}(t)\right\}_{t>0}$ as follows:

$$
S_{G}(t)=\beta^{-1} S_{S G}(\alpha t)
$$

We will refer to $\left\{S_{G}(t)\right\}_{i>0}$ as a Gamma $(\alpha, \beta)$ process, so that the standardised Gamma process, $\left\{S_{S G}(t)\right\}_{t>0}$, is a Gamma $(1,1)$ process. Note that the random variable $S_{G}(t)$ has a Gamma $(\alpha t, \beta)$ distribution. (Note also that we are parameterising the Gamma distribution so that $S_{G}(t)$ has mean $\alpha t / \beta$.)

The remainder of this section will be concerned with the standardised Gamma process. We are regarding this as a model for the aggregate claims process for a risk, so that $S_{S G}(t)$ represents the aggregate claims generated by this risk in the period $(0, t]$. We assume that premium income is received continuously at constant rate $c$ per unit time for this risk. We assume that

$$
c>E\left[S_{S G}(1)\right](=1)
$$

We denote by $\delta_{S G}(U, t)$ the probability of survival, i.e. non-ruin, up to time $t$ for this process given initial surplus $U(\geq 0)$, so that:

$$
\delta_{S G}(U, t)=\operatorname{Pr}\left(S_{S G}(\tau) \leq U+c \tau \text { for all } \tau, 0<\tau \leq t\right)
$$

The main result of this section is the following:

\section{Result:}

$$
\begin{aligned}
\delta_{S G}(0, t)=F_{S G}(c t, t)- & \frac{1}{c} F_{S G}(c t, t+1) \\
\delta_{S G}(U, t)=F_{S G}(U+c t, t) & -c \int_{0}^{t} f_{S G}(U+c s, s) F_{S G}(c(t-s), t-s) d s+ \\
& +\int_{0}^{t} f_{S G}(U+c s, s) F_{S G}(c(t-s), t-s+1) d s
\end{aligned}
$$


where $f_{S G}(x, t)$ and $F_{S G}(x, t)$ are the density function and distribution function, respectively, of a $\operatorname{Gamma}(t, 1)$ random variable.

Proof: For $n=1,2,3, \ldots$ and $U \geq 0$ define:

$$
\delta(U, t ; n)=\operatorname{Pr}(S(\tau ; n) \leq U+c \tau \text { for all } \tau, 0<\tau \leq t)
$$

The first step in the proof of this result is to show that:

$$
\operatorname{Lim}_{n \rightarrow \infty} \delta(U, t ; n)=\delta(U, t)
$$

To see this, note from the construction of the processes $\left\{\{S(t ; n)\}_{t>0}\right\}$ and from (2.2) that for any sample path $\omega$ and any $\tau \in(0, t]$ :

$$
\ldots S(\tau ; n-1)(\omega) \leq S(\tau ; n)(\omega) \leq \ldots \leq S_{S G}(\tau)(\omega)
$$

and:

$$
\operatorname{Lim}_{n \rightarrow \infty} S(\tau ; n)(\omega)=S_{S G}(\tau)(\omega)
$$

Hence :

$$
\operatorname{Lim}_{n \rightarrow \infty} \delta(U, t ; n) \geq \delta(U, t)
$$

and the limit on the left does exist. If this limit is strictly greater than $\delta(U, t)$, then there must exist some sample path $\omega$ and some $\tau \in(0, t]$ such that for all $n$ :

$$
S(\tau ; n)(\omega) \leq U+c \tau<S_{S G}(\tau)(\omega)
$$

which contradicts (2.6). This proves (2.5).

Since $\{S(t ; n)\}_{t>0}$ is a compound Poisson process, we have:

$$
\delta(0, t ; n)=\frac{1}{c t} \int_{0}^{c t} F(y, t ; n) d y
$$

where $F(y, t ; n)$ is the distribution function of $S(t ; n)$. See SEAL [1978b, Ch. 4]. The convergence in distribution of the processes $\left\{\{S(t ; n)\}_{t>0}\right\}$ and the fact that $F_{S G}(y, t)$ is everywhere continuous show that for all $y$ and all $t$ :

$$
F_{S G}(y, t)=\operatorname{Lim}_{n \rightarrow \infty} F(y, t ; n)
$$

Applications of this result, of (2.5) and of the Bounded Convergence Theorem to (2.7) show that:

$$
\delta_{S G}(0, t)=\frac{1}{c t} \int_{0}^{c t} F_{S G}(y, t) d y
$$


Since $S_{S G}(t)$ has a Gamma $(t, 1)$ distribution, we can rewrite (2.8) as follows:

$$
\begin{aligned}
\delta_{S G}(0, t) & =\frac{1}{c t} \int_{0}^{c t} \int_{0}^{x} \frac{y^{t-1} e^{-y}}{\Gamma(t)} d y d x \\
& =\frac{1}{c t} \int_{0}^{c t} \int_{y}^{c t} \frac{y^{t-1} e^{-y}}{\Gamma(t)} d x d y \\
& =\int_{0}^{c t} \frac{y^{t-1} e^{-y}}{\Gamma(t)} d y-\frac{\Gamma(t+1)}{c t \Gamma(t)} \int_{0}^{c t} \frac{y^{t} e^{-y}}{\Gamma(t+1)} d y \\
& =F_{S G}(c t, t)-\frac{1}{c} F_{S G}(c t, t+1)
\end{aligned}
$$

This proves (2.3). Formula (2.4) can be derived as follows. Using the familiar general reasoning argument (see SEAL [1974, p. 126]), we have:

$$
\delta_{S G}(U, t)=F_{S G}(U+c t, t)-c \int_{0}^{t} \delta_{S G}(0, t-s) f_{S G}(U+c s, s) d s
$$

Formula (2.4) is obtained by substituting (2.3) into this last expression.

Table 1 shows values of $\delta_{S G}(U, t)$ for the standardised Gamma process for various combinations of $U$ and $t$. The premium has been taken to be 1.1 per unit time, so that the premium loading factor is $10 \%$.

TABLE 1

VALUES OF $\delta_{S G}(U, t)$ WHEN $c=1.1$

\begin{tabular}{rlllll}
\hline$t$ & $U=0$ & $U=1$ & $U=5$ & $U=10$ & $U=25$ \\
\hline 1 & 0.39352 & 0.82211 & 0.99706 & 0.99998 & 1.00000 \\
2 & 0.30244 & 0.71818 & 0.99108 & 0.99991 & 1.00000 \\
3 & 0.25906 & 0.65070 & 0.98304 & 0.99975 & 1.00000 \\
4 & 0.23254 & 0.60271 & 0.97378 & 0.99947 & 1.00000 \\
5 & 0.21423 & 0.56647 & 0.96393 & 0.99907 & 1.00000 \\
6 & 0.20064 & 0.53790 & 0.95386 & 0.99852 & 1.00000 \\
7 & 0.19005 & 0.51467 & 0.94384 & 0.99784 & 1.00000 \\
8 & 0.18151 & 0.49532 & 0.93402 & 0.99703 & 1.00000 \\
9 & 0.17445 & 0.47890 & 0.92449 & 0.99608 & 1.00000 \\
10 & 0.16848 & 0.46475 & 0.91531 & 0.99503 & 1.00000 \\
20 & 0.13662 & 0.38506 & 0.84296 & 0.98058 & 0.99999 \\
30 & 0.12306 & 0.34924 & 0.79653 & 0.96428 & 0.99996 \\
40 & 0.11534 & 0.32837 & 0.76483 & 0.94935 & 0.99987 \\
50 & 0.11030 & 0.31460 & 0.74194 & 0.93644 & 0.99971 \\
100 & 0.09919 & 0.28380 & 0.68487 & 0.89569 & 0.99801 \\
200 & 0.09351 & 0.26783 & 0.65187 & 0.86585 & 0.99409 \\
300 & 0.09195 & 0.26345 & 0.64234 & 0.85617 & 0.99176 \\
400 & 0.09138 & 0.26182 & 0.63872 & 0.85233 & 0.99055 \\
500 & 0.09113 & 0.26112 & 0.63717 & 0.85063 & 0.98993 \\
600 & 0.09102 & 0.26080 & 0.63645 & 0.84983 & 0.98961 \\
700 & 0.09097 & 0.26065 & 0.63610 & 0.84944 & 0.98945 \\
800 & 0.09094 & 0.26057 & 0.63592 & 0.84924 & 0.98936 \\
900 & 0.09093 & 0.26053 & 0.63582 & 0.84913 & 0.98931 \\
1000 & 0.09092 & 0.26051 & 0.63578 & 0.84908 & 0.98928 \\
& & & & & \\
\hline & & & & & \\
\hline
\end{tabular}




\section{3. the Gamma process approximation to a COMpound Poisson process}

In this section we demonstrate how finite time survival probabilities for a compound Poisson process can be approximated by those for a Gamma process.

Let $\{S(t)\}_{t>0}$ be a compound Poisson process with Poisson parameter $\lambda$. Let $P(x)$ denote the individual claim amount distribution and let $p_{k}$ denote the $k$-th moment about zero of this distribution. We shall approximate this compound Poisson process by a Gamma $(\alpha, \beta)$ process $\left\{S_{G}(t)\right\}_{t>0}$. We find the parameters $\alpha$ and $\beta$ of the Gamma process by matching the first two moments of the two processes (assuming that these moments exist). For each value of $t>0$ we set

$$
\begin{aligned}
& E[S(t)]=\lambda t p_{1}=\alpha t / \beta=E\left[S_{G}(t)\right] \\
& V[S(t)]=\lambda t p_{2}=\alpha t / \beta^{2}=V\left[S_{G}(t)\right]
\end{aligned}
$$

which gives

$$
\beta=p_{1} / p_{2} \quad \text { and } \quad \alpha=\lambda p_{1}^{2} / p_{2}
$$

Note that the parameters $\alpha$ and $\beta$ are independent of $t$.

The surplus process associated with the compound Poisson process is $\{U(t)\}_{t>0}$ where $U(t)=U+c t-S(t) . U$ is the initial surplus and $c$ is the premium income per unit time. In our numerical examples we write $c=(1+\theta) \lambda p_{1}$ where $\theta$ is the premium loading factor. The finite time survival probability for this process is $\delta(U, t)$ defined by

$$
\delta(U, t)=\operatorname{Pr}(S(\tau) \leq U+c \tau \text { for all } \tau, 0<\tau \leq t)
$$

We will approximate this probability by $\delta_{G}(U, t)$ defined by

$$
\delta_{G}(U, t)=\operatorname{Pr}\left(S_{G}(\tau) \leq U+c \tau \text { for all } \tau, 0<\tau \leq t\right)
$$

where the parameters $\alpha$ and $\beta$ of $\left\{S_{G}(t)\right\}_{t>0}$ are given by (3.1). We calculate $\delta_{G}(U, t)$ using the standardised Gamma process and the identity

$$
\begin{aligned}
\delta_{G}(U, t) & =\operatorname{Pr}\left(S_{G}(\tau) \leq U+(1+\theta) \alpha \tau / \beta \text { for all } \tau, 0<\tau \leq t\right) \\
& =\operatorname{Pr}\left(\beta^{-1} S_{S G}(\tau) \leq U+(1+\theta) \alpha \tau / \beta \text { for all } \tau, 0<\tau \leq t\right) \\
& =\operatorname{Pr}\left(S_{S G}(\alpha \tau) \leq \beta U+(1+\theta) \tau \text { for all } \tau, 0<\tau \leq \alpha t\right) \\
& =\delta_{S G}(\beta U, \alpha t)
\end{aligned}
$$

In our numerical examples we set $\lambda=1$ and use two distributions for individual claims amounts :

Distribution 1: $P(x)$ is the Gamma $(1 / 3,1 / 3)$ distribution. For this distribution, $p_{1}=1$ and $p_{2}=4$, so that $\alpha=\beta=0.25$ in the approximating gamma process. Thus we approximate $\delta(U, t)$ by $\delta_{S G}(U / 4, t / 4)$.

Distribution 2: $P(x)$ is the Pareto $(3,2)$ distribution. For this distribution, $p_{1}=1$ and $p_{2}=4$, so that $\alpha=\beta=0.25$ in the approximating Gamma process.

The parameters of these distributions have been chosen so that the same Gamma process approximates each compound Poisson process. Although the 
first two moments are the same for each of Distributions 1 and 2, $p_{3}$ does not exist for Distribution 2, but does exist for Distribution 1. Hence we would expect that the Gamma process approximation would be better for the compound Poisson process with Distribution 1 rather than Distribution 2.

In Figures 1 to 5 the crosses denote the ratios $\delta(U, t): \delta_{S G}(\beta U, \alpha t)$ (shown as percentages) for selected values of $U$ and $t$ for the surplus process associated with Distribution 1 when $\theta=0.1$. A logarithmic scale for time has been used in these graphs. The values of $\delta(U, t)$ have been calculated (approximately) using the methods described by DICKSON and WATERS [1991]. We note that for each of these values of $U$ the approximate values are fairly close to the true values. They are within $4 \%$ of the true values for all values of $U$ shown, and within $1 \%$ for virtually all combinations of $U$ and $t$, except when $U=4$. We conclude that the approximation to $\delta(U, t)$ is reasonable in this case.

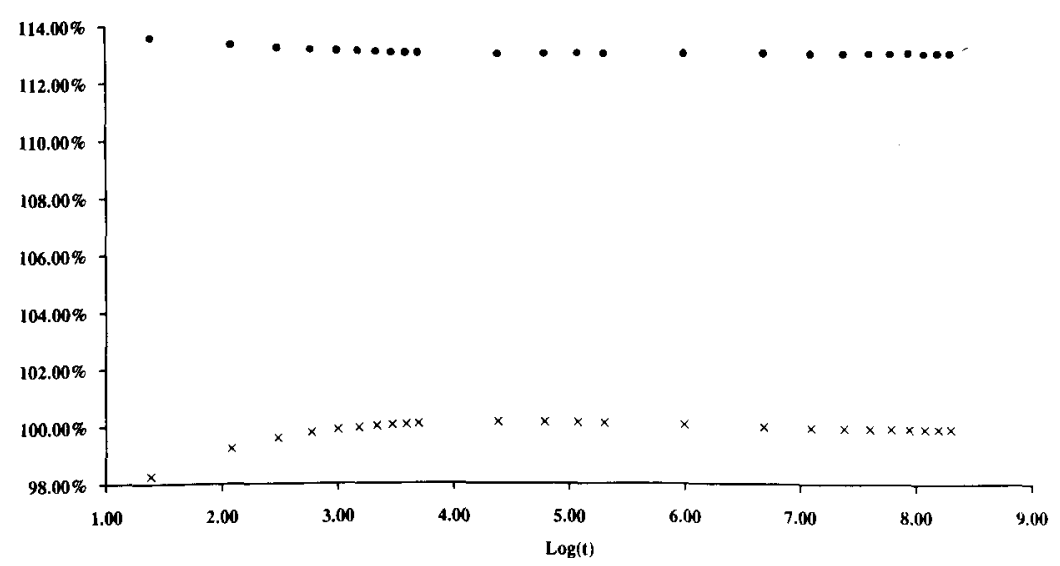

FigURE 1. Ratios of exact to approximate values of $\delta(0, t)$ for Gamma individual claims.

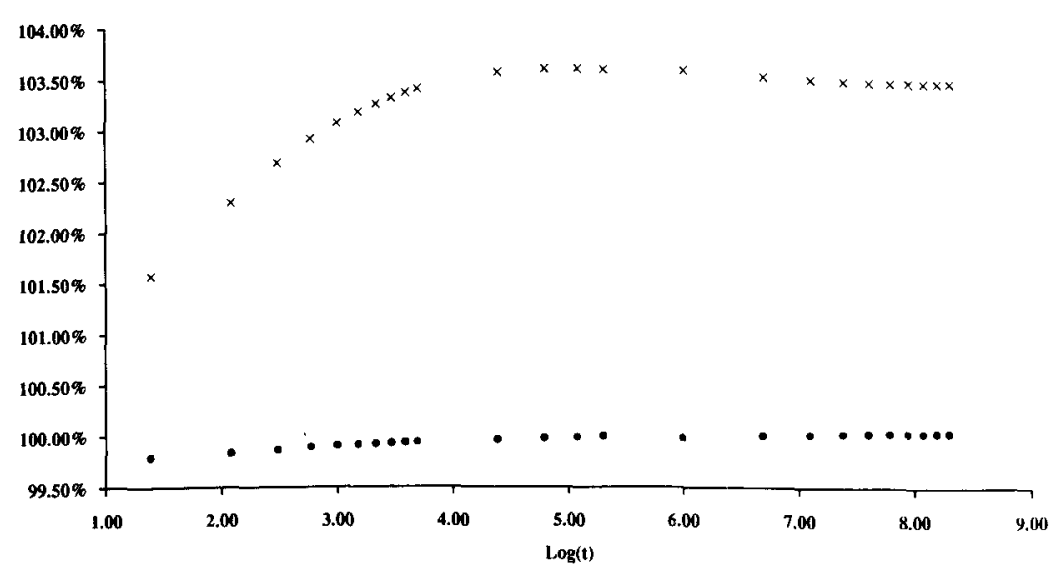

FIgURE 2. Ratios of exact to approximate values of $\delta(4, t)$ for Gamma individual claims. 


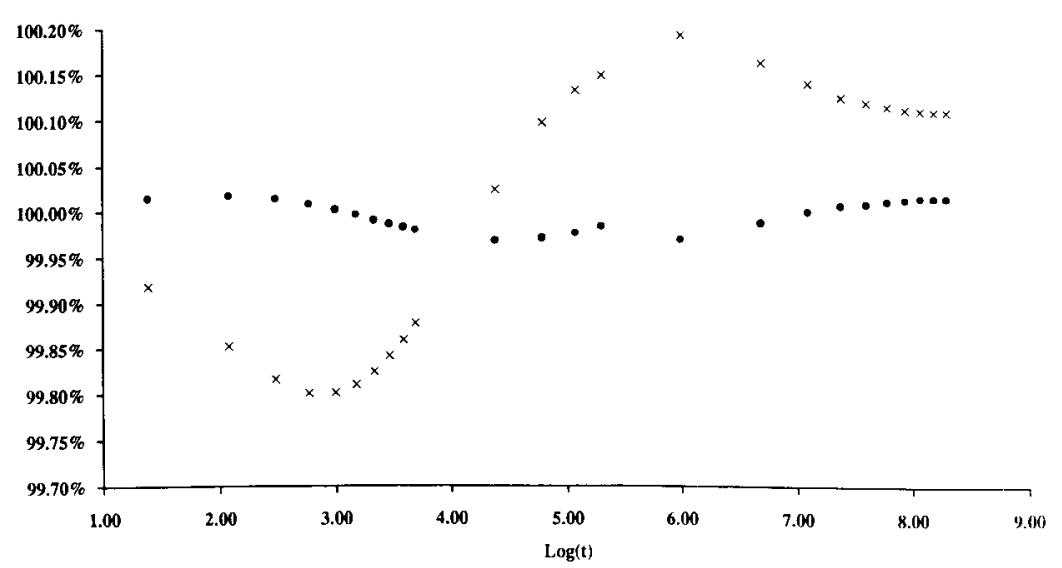

Figure 3. Ratios of exact to approximate values of $\delta(20, t)$ for Gamma individual claims.

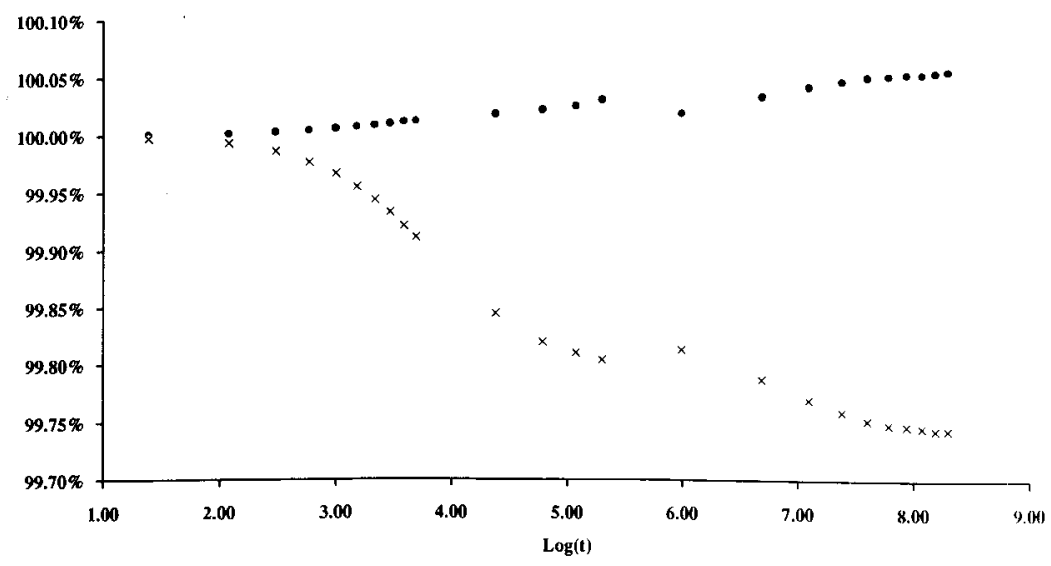

FIGURE 4. Ratios of exact to approximate values of $\delta(40, t)$ for Gamma individual claims.

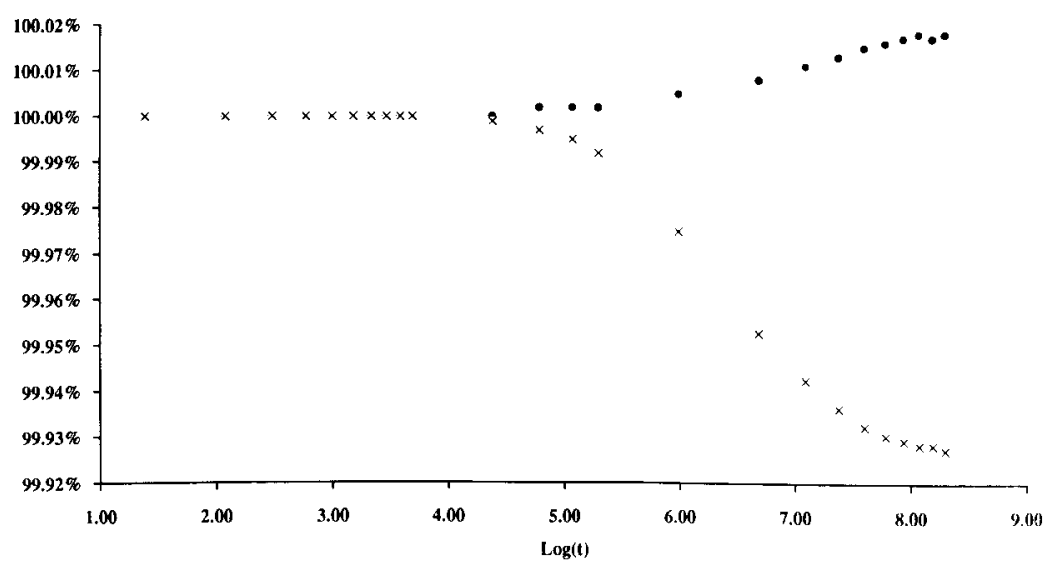

FIGURE 5. Ratios of exact to approximate values of $\delta(100, t)$ for Gamma individual claims. 
The crosses in Figures 6 to 10 denote the corresponding ratios for the same combinations of $U$ and $t$ for the surplus processs associated with Distribution 2, again with $\theta=0.1$ and with a logarithmic scale for time. The approximations are generally worse for this distribution, although the approximate values are within $1 \%$ of the true values when $U=40$ and when $U=100$.

We have used the same Gamma process to approximate to two different compound Poisson processes. It is therefore no surprise that the approximation is better for one of the compound Poisson processes. In the following section we present a method which allows us to approximate survival probabilities for compound Poisson processes with identical first two moments by different Gamma processes.

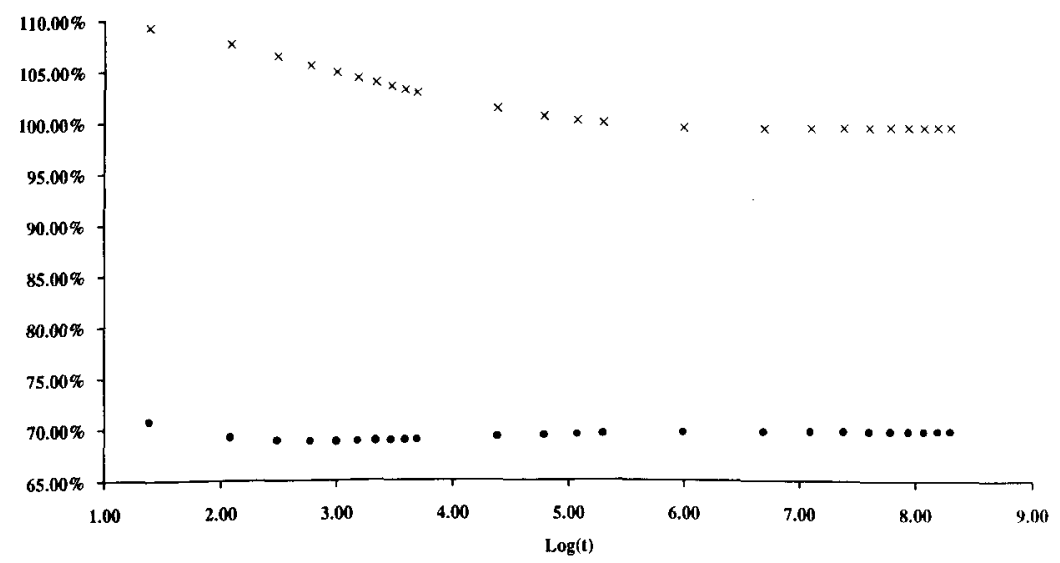

Figure 6. Ratios of exact to approximate values of $\delta(0, t)$ for Pareto individual claims.

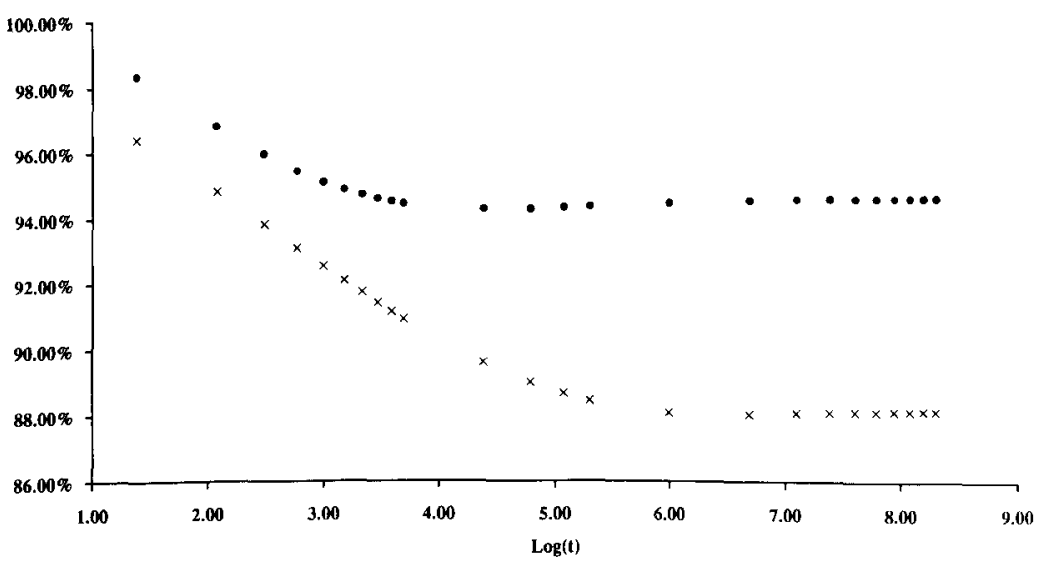

FigURE 7. Ratios of exact to approximate values of $\delta(4, t)$ for Pareto individual claims. 


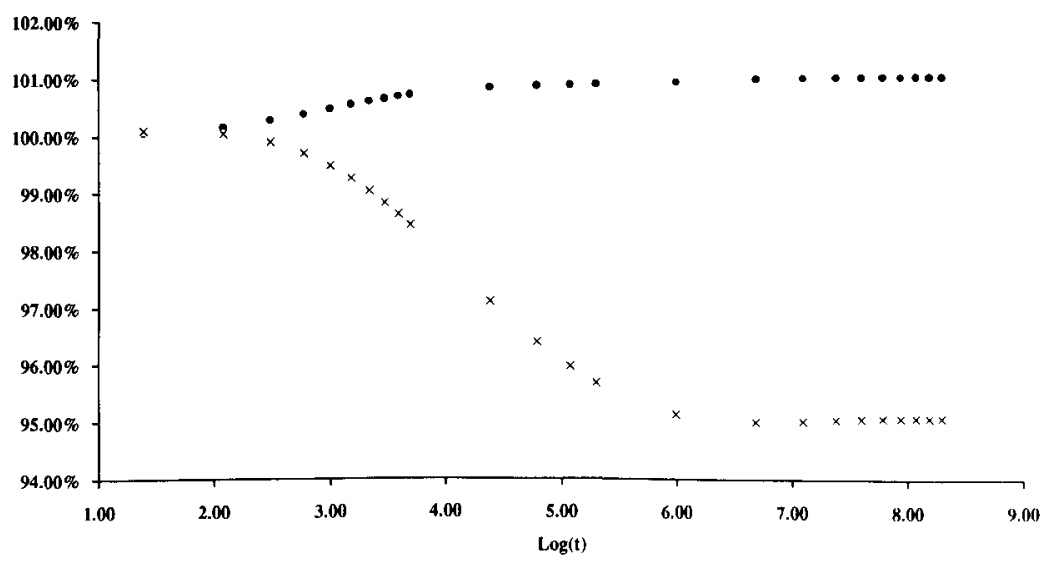

FIGURE 8. Ratios of exact to approximate values of $\delta(20, t)$ for Pareto individual claims.

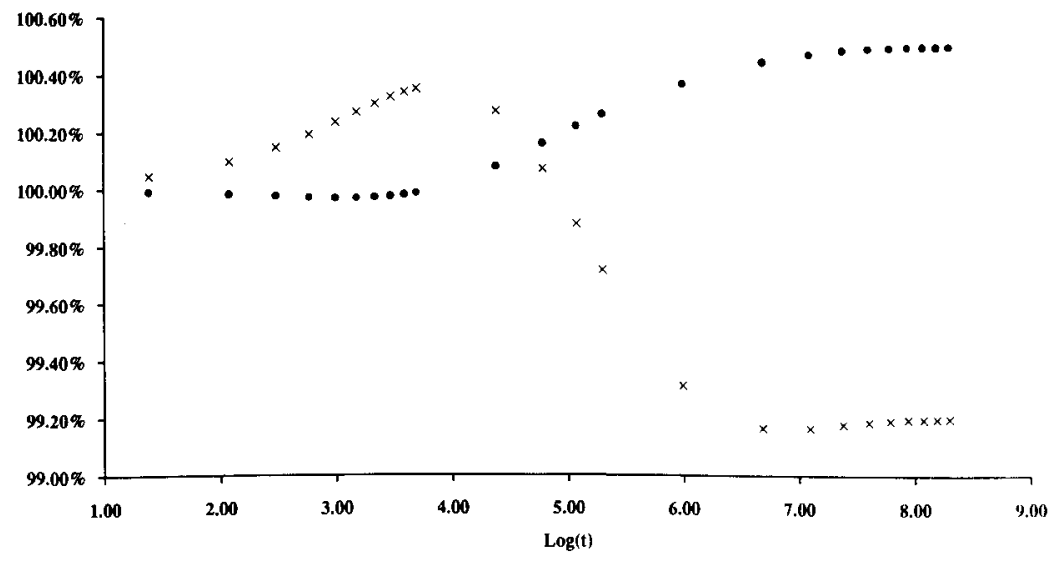

FIGURE 9. Ratios of exact to approximate values of $\delta(40, t)$ for Pareto individual claims.

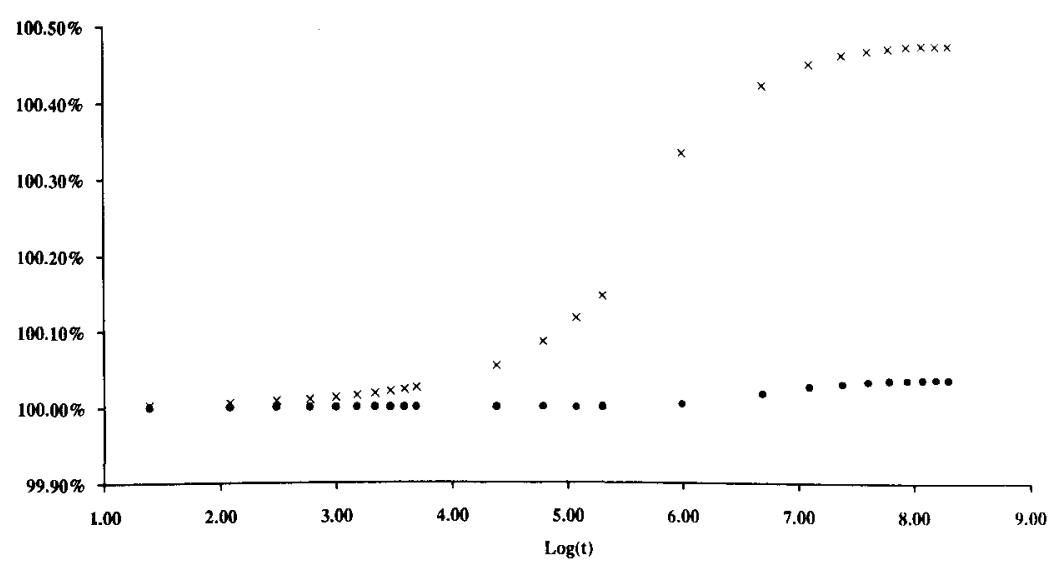

Figure 10. Ratios of exact to approximate values of $\delta(100, t)$ for Pareto individual claims. 


\section{THE TRANSLATED GAMMA PROCESS APPROXIMATION TO A COMPOUND POISSON PROCESS}

As in the previous section, $\{S(t)\}_{t>0}$ is a compound Poisson process with Poisson parameter $\lambda ; p_{k}$ denotes the $k$-th moment about zero of the individual claim amount distribution. We shall approximate $\{S(t)\}_{t>0}$ by what we term a translated Gamma process $\left\{S_{T G}(t)\right\}_{t>0}$. For all $t>0$ we define

$$
S_{T G}(t)=S_{G}(t)+k t
$$

where $\left\{S_{G}(t)\right\}_{t>0}$ is a $\operatorname{Gamma}(\alpha, \beta)$ process and $k$ is some constant (which may be positive or negative). The parameters $\alpha, \beta$ and $k$ of the process $\left\{S_{T G}(t)\right\}_{t>0}$ are chosen such that for all $t>0$

$$
\begin{aligned}
& E[S(t)]=\lambda t p_{1}=\alpha t / \beta+k t=E\left[S_{T G}(t)\right] \\
& V[S(t)]=\lambda t p_{2}=\alpha t / \beta^{2}=V\left[S_{T G}(t)\right] \\
& S k[S(t)]=\lambda t p_{3} /\left(\lambda t p_{2}\right)^{3 / 2}=2 /(\alpha t)^{1 / 2}=S k\left[S_{T G}(t)\right]
\end{aligned}
$$

i.e. we are matching the mean, variance and coefficient of skewness of $S(t)$ and $S_{T G}(t)$ for all $t$ (again assuming that these quantities exist). These identities give the parameter values as

$$
\alpha=4 \lambda p_{2}^{3} / p_{3}^{2} \quad \beta=2 p_{2} / p_{3} \quad k=\lambda\left(p_{1}-2 p_{2}^{2} / p_{3}\right)
$$

As in the previous approximation, the parameters are all independent of $t$. We now approximate

by

$$
\delta(U, t)=\operatorname{Pr}(S(\tau) \leq U+c \tau \text { for all } \tau, 0<\tau \leq t)
$$

$$
\begin{aligned}
\delta_{T G}(U, t) & =\operatorname{Pr}\left(S_{T G}(\tau) \leq U+c \tau \text { for all } \tau, 0<\tau \leq t\right) \\
& =\operatorname{Pr}\left(S_{G}(\tau)+k \tau \leq U+c \tau \text { for all } \tau, 0<\tau \leq t\right) \\
& =\operatorname{Pr}\left(S_{G}(\tau) \leq U+(c-k) \tau \text { for all } \tau, 0<\tau \leq t\right)
\end{aligned}
$$

Note that

$$
\begin{aligned}
c-k & =(1+\theta) \lambda p_{1}-\lambda\left(p_{1}-2 p_{2}^{2} / p_{3}\right) \\
& =\theta \lambda p_{1}+2 \lambda p_{2}^{2} / p_{3} \\
& =\theta \lambda p_{1}+\alpha / \beta
\end{aligned}
$$

Thus $c-k>E\left[S_{G}(1)\right]$ (where $S_{G}(1)$ has a Gamma $(\alpha, \beta)$ distribution) regardless of the values of $k$. Since $\lambda p_{1}=\alpha / \beta+k, \delta_{T G}(U, t)$ represents the finite time survival probability when the aggregate claims process is a $\operatorname{Gamma}(\alpha, \beta)$ process and when the premium loading factor is $\hat{\theta}=\theta(1+k \beta / \alpha)$. Hence we can approximate $\delta(U, t)$ by $\delta_{S G}(\beta U, \alpha t)$ using a premium loading factor of $\hat{\theta}$.

We illustrate the approximation method for two distributions for individual claim amounts:

Distribution 1: $P(x)$ is the Gamma $(1 / 3,1 / 3)$ distribution. The first three moments of this distribution are $p_{1}=1, p_{2}=4$ and $p_{3}=28$. Let $\lambda=1$ and let $\theta=0.1$, giving $\alpha=16 / 49, \beta=2 / 7$ and $k=-1 / 7$. Then $\alpha / \beta=8 / 7$ and $c-k=87 / 70$, so that $\hat{\theta}=7 / 80$. We approximate $\delta(U, t)$ by $\delta_{S G}(2 U / 7,16 t / 49)$ using a premium loading factor of $7 / 80$. 
In Figures 1 to 5 the circles denote the ratios $\delta(U, t): \delta_{S G}(\beta U, \alpha t)$ for the same values of $U$ and $t$ as before for the surplus process associated with Distribution 1. Again a logarithmic scale has been used for time. The approximate values are very close to the exact ones except when $U=0$ (within $0.3 \%$ when $U=4$, and within $0.06 \%$ when $U=20,40$ and 100 ). It is no surprise that the translated Gamma process gives superior approximations to those in the previous section when $U>0$ since the approximating process matches one further feature of the given process. The reason why the approximation is poor when $U=0$ is discussed later in this section.

Distribution 3: $P(x)$ is the Pareto $(4,3)$ distribution. (Note that the third moment of Distribution 2 does not exist and so we cannot use the translated Gamma approximation in this case.) For this distribution $p_{1}=1, p_{2}=3$ and $p_{3}=27$. Again let $\lambda=1$ and let $\theta=0.1$ giving $\alpha=4 / 27, \beta=2 / 9$ and $k=1 / 3$. Then $\alpha / \beta=2 / 3$ and $c-k=23 / 30$, so that $\hat{\theta}=0.15$. Hence we approximate $\delta(U, t)$ by $\delta_{S G}(2 U / 9,4 t / 27)$ using a premium loading factor of 0.15 . In Figures 6 to 10 the circles denote the ratios $\delta(U, t): \delta_{S G}(\beta U, \alpha t)$ for the same values of $U$ and $t$ as before for the surplus process associated with Distribution 3. The approximate values are fairly close to the exact ones when $U>0$ (within $6 \%$ when $U=4$, and within about $1 \%$ when $U=20,40$ and 100 ) but the approximations are not as close as for Distribution 1. Note that the ratios are not directly comparable with those for Distribution 2 as the parameter values for the distributions are different. Nevertheless, when $U>0$ this approximation represents an improvement over the method described in the preceding section.

Since the first three moments of the approximating translated gamma process match those of the compound Poisson process for all values of $t$, we would expect $\delta_{T G}(U, t)$ to be reasonable approximation to $\delta(U, t)$. This is indeed the case when $U>0$. However, when $U=0$, the approximations are poor and are much worse than those in the previous section. We can see why this is so by writing down formulae for the exact and approximate survival probabilities. The formula for the exact survival probability is

$$
\delta(0, t)=\frac{1}{c t} \int_{0}^{c t} F(x, t) d x
$$

where $F(x, t)=\operatorname{Pr}(S(t) \leq x)$, and $S(t)$ has a compound Poisson distribution. The approximation in Section 3 is

$$
\delta_{G}(U, t)=\frac{1}{c t} \int_{0}^{c t} F_{G}(x, t) d x
$$

where $F_{G}(x, t)=\operatorname{Pr}\left(S_{G}(t) \leq x\right)$, and $S_{G}(t)$ has a Gamma distribution whose first two moments match those of the compound Poisson distribution. It is not surprising that (4.2) is a good approximation to (4.1). Not only are the formulae for $\delta(0, t)$ and $\delta_{G}(0, t)$ of the same form, but $F_{G}(x, t)$ may be 
regarded as an intuitively reasonable approximation to $F(x, t)$ since these two distributions have the same first two moments.

In this section $\delta(0, t)$ is approximated by

$$
\delta_{T G}(0, t)=\frac{1}{(c-k) t} \int_{0}^{(c-k) t} F_{G}(x, t) d x
$$

Comparing (4.1) and (4.3), we see that there are two differences: the factor $c$ in (4.1) is replaced by $c-k$ in (4.3), and $F_{G}(x, t)$ does not have the same first two moments as $F(x, t)$. It is not surprising that (4.3) is not as good an approximation to (4.1) as is (4.2). In particular, in the limiting case when $t \rightarrow \infty$ we can measure the difference between (4:1) and (4.3) since

$$
\operatorname{Lim}_{t \rightarrow \infty} \delta(0, t)=1 /(1+\theta) \quad \text { and } \quad \operatorname{Lim}_{t \rightarrow \infty} \delta_{T G}(0, t)=1 /(1+\hat{\theta})
$$

(See Dufresne et al. [1991].)

\section{CONCLUDING REMARKS}

The computer time required to produce approximate values for $\delta(U, t)$ using the methods of the previous two sections is substantially less than that required for the algorithms described by Dickson and WATERs [1991]. Although these algorithms produce very accurate values, the amount of computer time required for large values of $U$ and $t$ can be considerable. The examples in Section 4 show that the approximations to $\delta(U, t)$ are good for large values of $U$ and $t$. We conclude that the approximation method of the previous section can be used to produce fast and fairly reliable estimates of $\delta(U, t)$ for such combinations of $U$ and $t$.

The underlying idea in Sections 3 and 4 has been to approximate a compound Poisson process $\{S(t)\}_{t>0}$ by a Gamma process $\left\{S_{G}(t)\right\}_{t>0}$ and by a translated Gamma process $\left\{S_{T G}(t)\right\}_{t>0}$, respectively. In each case the approximation is the result of matching an appropriate number of moments. The probability of survival for the compound Poisson process, $\delta(U, t)$, is then approximated by the corresponding probability for the Gamma process or the translated Gamma process. However, there is an alternative and related way of approximating this probability. We have the following formulae:

$$
\begin{gathered}
\delta(0, t)=\frac{1}{c t} \int_{0}^{c t} F(x, t) d x \\
\delta(U, t)=F(U+c t, t)-c \int_{0}^{t} \delta(0, t-s) f(U+c s, s) d s
\end{gathered}
$$

(Formula (5.1) is, of course, the same as formula (4.1).)

We can now approximate $\delta(0, t)$ and $\delta(U, t)$ by approximating $F(\cdot, t)$ and $f(\cdot, s)$ by Gamma distributions or translated Gamma distributions with the 
same first two or three moments as the original distributions. If we approximate $F(\cdot, t)$ and $f(\cdot, s)$ by Gamma distributions, it can be checked that the result is the same as that achieved by approximating the original compound Poisson process by a Gamma process, as in Section 3. However, replacing $F(\cdot, t)$ and $f(\cdot, s)$ in (5.1) and (5.2) by translated Gamma distributions with the same first three moments, an idea originally discussed by SEAL [1978a], is not the same as approximating the original process by a translated Gamma process as described in Section 4. To see this, note that Seal's method leads to the following approximation for $\delta(0, t)$ :

$$
\delta(0, t) \approx \frac{1}{c t} \int_{0}^{c t} F_{T G}^{*}(x, t) d x
$$

where $F_{T G}^{*}(x, t)$ is a translated Gamma distribution with the same first three moments as $F(x, t)$. Formula (5.3) is clearly not the same as formula (4.3). An advantage of Seal's method as compared to the method of Section 4 is that (5.3) is a better approximation to (4.1) than is (4.3). A disadvantage of Seal's method is that it leads to a slightly more complicated (approximate) formula for $\delta(0, t)$ and hence for $\delta(U, t)$.

\section{REFERENCES}

Dickson, D.C.M. and Waters, H.R. (1991) Recursive calculation of survival probabilities. ASTIN Bulletin 22, 199--221.

Dufresne, F., Gerber, H.U. and Shiu, E.S.W. (1991) Risk theory and the gamma process. ASTIN Bulletin 22, 177-192.

SEAL, H. L. (1974) The numericl calculation of $U(w, t)$, the probability of non-ruin in an interval $(0, t)$. Scandinavian Acutarial Journal, 121-139.

SEAL, H. L. (1978a) From aggregate claims distribution to probability of ruin. ASTIN Bulletin 10. $47-53$.

SEAL, H.L. (1978b) Survival probabilities: the goal of risk theory. John Wiley and Sons, New York.

David C. M. Dickson

Centre for Actuarial Studies,

University of Melbourne,

Parkville,

Victoria 3052,

Australia
HOWARD R. WATERS

Department of Actuarial Mathematics

and Statistics,

Heriot-Watt University,

Edinburgh EH14 4AS,

Scotland 